\title{
Acompañamiento socioemocional en pandemia: tutorías PACE a estudiantes de cuarto año medio
}

\author{
Socio-Emotional Accompaniment in Pandemic: PACE Tutoring for Fourth \\ Year High School Students
}

Milena Vásquez-Villegas ${ }^{1}$

\begin{abstract}
RESUMEN
El acompañamiento socioemocional es una de las áreas identificadas por la Unesco como fundamentales a desarrollar en el ámbito educativo, en el contexto de la pandemia por COVID-19 durante el año 2020. El Área de Gestión Personal del Programa de Acompañamiento y Acceso Efectivo a la Educación Superior (PACE) de la Universidad Austral de Chile (UACh), sede Puerto Montt, tiene como principal objetivo abordar el desarrollo socioemocional y vocacional de estudiantes en su etapa de preparación para la universidad. El presente artículo da a conocer las estrategias metodológicas de la implementación de acciones que permitieron que el Área de Gestión Personal de PACE-UACh cumpliera con las directrices propuestas por el Ministerio de Educación, para estudiantes de $4^{\circ}$ año medio en contexto virtual. Dentro de los principales hallazgos, se advierte el aporte realizado para la toma de decisiones, la ampliación de expectativas y la construcción de proyectos de vida, así como para la identificación de talentos y el proceso de autoconocimiento de las y los estudiantes. Por último, se destaca la importancia del acompañamiento al desarrollo socioemocional como un deber ético y se considera una adecuada conectividad como un derecho fundamental que debiese estar garantizado, para así cumplir con las necesidades educativas de la población en contexto de emergencia sanitaria.
\end{abstract}

Palabras claves: educación a distancia; educación superior; asesoramiento; desarrollo afectivo; pandemia; COVID-19.

\section{ABSTRACT}

Socioemotional accompaniment is one of the areas identified by UNESCO as fundamental to be developed in the educational field, in the context of the COVID-19 pandemic during 2020. The Personal Management Area of the Support and Effective Access to Higher Education Program (PACE) of the Universidad Austral of Chile (UACh), Puerto Montt, has as its main objective to address the socioemotional and vocational development of students in their preparatory stage for university. This

\footnotetext{
1 Terapeuta Ocupacional, área Gestión Personal del Programa PACE de la Universidad Austral de Chile, Puerto Montt, Chile; Magíster (c) en Estudios de Género e Intervención Psicosocial, Universidad Central, Chile; milena.vasquez.v@gmail.com.
} 
article presents the methodological strategies for the implementation of actions that allowed the Personal Management Area of PACE-UACh to comply with the guidelines proposed by the Ministry of Education, for 4th year high school students in a virtual context. Among the main findings is the contribution for decision-making, expansion of expectations and construction of life projects, as well as for the identification of talents and the process of self-knowledge of the students. Finally, the importance of accompanying socioemotional development is highlighted as an ethical duty and an adequate connectivity is indicated as a fundamental right that should be guaranteed, in order to meet the educational needs of the population in the context of a health emergency.

Keywords: Distance education; higher education; counseling; affective development; pandemic; COVID-19.

\section{Introducción}

El acceso a la educación superior en Chile, hasta hace pocos años, dependía exclusivamente del puntaje que cada estudiante pudiese obtener en el examen nacional estandarizado, llamado Prueba de Selección Universitaria (PSU), el cual evidenciaba la desigualdad socioeconómica -y consecuentemente académica- existente entre los establecimientos educacionales municipales y particulares pagados del país.

Durante las últimas décadas se han incorporado nuevas vías de ingreso universitario, entre ellas el Programa de Acompañamiento y Acceso Efectivo a la Educación Superior (PACE), el cual surge en 2014 con el objetivo de restituir el derecho a la educación superior a estudiantes de establecimientos educacionales definidos como vulnerables por el Ministerio de Educación. El área de "Preparación en la Enseñanza Media" (PEM) de cada una de las 31 instituciones de educación superior en convenio, desarrolla actividades durante los últimos 2 años de enseñanza media, en distintos liceos patrocinados, permitiendo a los/as las estudiantes que egresan acceder a los cupos PACE dispuestos por cada universidad, luego de cumplir los requisitos de habilitación.

Lo anterior busca fortalecer la educación pública, aumentar la equidad, diversidad y calidad en la educación superior, implementando acciones que permitan reforzar contenidos académicos, así como promover la exploración vocacional de los y las estudiantes, 
desarrollando un acompañamiento socioemocional, con miras al ingreso, mantención y titulación en la educación superior (Ministerio de Educación [Mineduc], s.f.).

El proceso de acompañamiento a estudiantes de $4^{\circ}$ año medio llevado a cabo en la Universidad Austral de Chile (UACh), sede Puerto Montt, recibe el nombre de "Preparación Académica Temprana" (PAT) y se implementa desde el año 2017, en un formato de clases presenciales durante 15 sesiones efectuadas los días sábado, en dependencias de la Universidad. Allí los y las estudiantes asisten a clases de Lengua y Literatura, Matemáticas y Gestión Personal.

En concordancia con lo propuesto por el Ministerio de Educación en sus planes y programas para la asignatura de Orientación (Ministerio de Educación, s.f.) y de acuerdo con los lineamientos del Plan de Exploración Vocacional perteneciente a la Programación Operativa PACE-UACh, es el área de Gestión Personal la que desarrolla el objetivo de "fortalecer en jóvenes el desarrollo de habilidades intra e interpersonales, ampliación de expectativas, clarificación vocacional y creación de un proyecto de vida, desde la óptica de los territorios en que opera el PACE-UACh"2. Lo anterior se trabaja mediante la construcción de representaciones positivas en las/os estudiantes, que incorporen sus características, motivaciones, intereses, habilidades y competencias, acompañando la construcción de un proyecto de vida basado en el conocimiento de lo anterior.

De este modo y hasta el año 2019, el área de Gestión Personal, en las actividades de "Preparación Académica Temprana" cumplía sus objetivos mediante la ejecución de un trabajo presencial, que favorecía el vínculo de los y las estudiantes con la educación superior. Allí, los/as estudiantes conocían la infraestructura, el funcionamiento y la oferta académica de su universidad patrocinante, así como también interactuaban con estudiantes de otros establecimientos, en grupos mixtos que congregaban a más de treinta personas cada uno. En estos espacios tenía lugar el análisis, la reflexión y se extraían conclusiones respecto a su proceso de autoconocimiento, exploración vocacional y toma de decisiones.

\footnotetext{
2 Fuente derivada de documentos internos de PACE-UACh (no accesible públicamente).
} 
De esta manera, la instancia presencial, que incluía la visita constante de los y las estudiantes a la universidad, se transformó en sí misma en una experiencia altamente valorada por ellos/as y, por lo tanto, difícil de igualar en instancias no presenciales.

\section{Problemática}

La aparición de la pandemia por COVID-19 a principios de marzo, creó un escenario diverso y complejo al congelar todas las propuestas y planificaciones anuales desarrolladas hasta el momento en las instituciones de educación media y superior. La cuarentena decretada por algunos municipios y la decisión de los planteles educativos de suspender las actividades presenciales se transformó en una realidad no exenta de polémicas, en constante tensión con los/as docentes, la ciudadanía, el Ministerio de Educación y el Ministerio de Salud.

Lo anterior obligó a muchas instituciones de educación a plantearse por primera vez el establecer una modalidad de trabajo que no incluyera clases presenciales, incorporando modalidades sincrónicas o asincrónicas en forma remota, con uso de herramientas digitales.

Dicha modificación puso en evidencia las condiciones de vulnerabilidad que experimentan los y las estudiantes pertenecientes a establecimientos educacionales PACE, donde destacan la pobreza, alta ruralidad y consecuente escasa conectividad por razones geográficas y materiales. En este nuevo contexto, muchos y muchas estudiantes se ven imposibilitados/as de acceder a la educación disponible en plataformas digitales, siendo en numerosas ocasiones los y las docentes quienes generan estrategias particulares para la resolución de esta problemática experimentada por sus educandos/as.

Desde diversas entidades educativas emerge una serie de recomendaciones y sugerencias pedagógicas que relevan la importancia del acompañamiento socioemocional a estudiantes en este contexto. Para Bisquerra y Pérez (2007) la educación emocional alude a procesos educativos continuos y permanentes, cuyo objetivo principal reside en el desarrollo de 
competencias emocionales que permitan a los/as estudiantes estar capacitados/as para la vida, aumentando su bienestar personal y social.

En este sentido, el Ministerio de Educación (2015) señala que la adquisición de estas competencias favorece el desarrollo del pensamiento crítico y, consecuentemente, una mejor y más informada toma de decisiones, por lo que promueve que los Estados parte integren el aprendizaje socioemocional en todos los niveles e instancias educativas.

En Chile, la fundación Educación 2020 realizó la encuesta Estamos Conectados, a partir de un cuestionario online con 23 preguntas, aplicado entre el 26 de marzo y el 17 de abril de 2020, con el objetivo de "conocer las condiciones y herramientas de las que disponen las comunidades educativas para sostener el proceso de enseñanza a distancia, recoger sus opiniones ante la pandemia (...) y apoyar en la toma de decisiones de las semanas que vienen” (Educación 2020, 2020). Esta encuesta contó con la participación de 3.340 personas, entre ellas estudiantes, apoderados/as, docentes y otros/as integrantes de las comunidades educativas, pertenecientes a establecimientos municipales, particulares subvencionados y privados, de diversas regiones del país.

En la encuesta, los/as estudiantes consultados/as reportaron su estado de ánimo más frecuente, identificándose un $63 \%$ de ellos/as con "aburrimiento", un $41 \%$ con "ansiedad o estrés", mientras que un 35\% manifestó experimentar "frustración y molestia". Por su parte, un $21 \%$ refirió sentir “tranquilidad” y solo un 3\% señaló estar "feliz" de encontrarse en casa. De los/as docentes encuestados/as, un 91,4\% manifiestó que el acompañamiento emocional hacia los y las estudiantes resulta más relevante que la enseñanza de contenidos académicos. Por último, respecto a las condiciones de conectividad por parte de los y las estudiantes, el $50 \%$ de ellas/os señaló tener acceso "ocasional" o "nulo" a un computador, mientras que un $45,7 \%$ reportó poseer solo un celular con internet.

Alejandra Arratia, directora ejecutiva de Educación 2020, sostiene que dichos resultados son "un llamado de alerta para repensar el valor que tienen las emociones en el proceso educativo y cómo profundizamos en la manera de abordarlas en la escuela, pero también desde la política pública” (Educación 2020, 2020). La autora señala la relevancia de revisar 
las condiciones estructurales del sistema educativo, de modo que estas permitan abordar el bienestar integral de niños, niñas y jóvenes, ya sea en este contexto de excepción como en condiciones de normalidad.

El Ministerio de Educación, en conjunto con Unesco y Unicef, se reunieron el mes de mayo de 2020 para realizar el seminario "Aprendizaje socioemocional en tiempos de pandemia" (Ministerio de Educación, 2020a), reforzando la importancia del desarrollo de dicha área y enfocando su abordaje al regreso de las clases presenciales. En este punto es importante detenerse, ya que llama la atención que el desarrollo socioemocional esté considerado, pero se encuentre supeditado al regreso a las clases presenciales, llamado que el Ministerio de Educación realizó insistentemente durante los primeros meses de emergencia sanitaria. Lo anterior implica eludir la responsabilidad de abordar el área socioemocional en un período clave como el que se está viviendo actualmente, aumentando el estrés y ansiedad de los/as estudiantes en el marco de una situación compleja y de gran incertidumbre.

A fines de mayo de 2020, el Ministerio de Educación pareció cambiar el foco, trasladándolo desde el retorno a las aulas hacia la entrega de recomendaciones a docentes y familias. Así, la Dirección de Educación General (Mineduc, 2020b) elaboró recursos de apoyo socioemocional, poniendo énfasis en la expresión y manejo de emociones por parte de los/as estudiantes, como una forma de contribuir al bienestar docente, la relación pedagógica actual y el bienestar de los/as integrantes del núcleo familiar.

Es en este punto donde resulta pertinente analizar cómo dichas recomendaciones y planteamientos teóricos se hacen realidad en una diversidad de contextos, especialmente en aquellos donde se ha vuelto evidente la desigualdad en el acceso a la educación y se han profundizado las brechas educativas y sociales ya existentes. Si las experiencias presenciales de vinculación de estudiantes de enseñanza media con la vida universitaria, propuestas por el Programa PACE hasta el 2019, ya no se pueden llevar a cabo de la misma forma, entonces cabe preguntarse: ¿cómo será posible la ampliación de expectativas en un escenario de nuevas vulneraciones?, ¿cómo resguardar y fortalecer los procesos de desarrollo socioemocional y vocacional en este nuevo contexto? 
Es por lo anterior, que el presente artículo tiene como principal objetivo sistematizar la experiencia de acompañamiento socioemocional realizada por el Área de Gestión Personal del Programa PACE-UACh, a estudiantes de $4^{\circ}$ medio de la Región de Los Lagos, durante la emergencia sanitaria. Para ello, se presentan las estrategias metodológicas adoptadas por el equipo, los resultados de impacto que ha tenido la iniciativa en términos de autoconocimiento y formulación de plan de vida, y el significado que los y las estudiantes le otorgan a este acompañamiento.

\section{Metodología}

\subsection{Diseño}

Durante el mes de abril de 2020 se realizó una revisión del Plan Operativo PACE-UACh, donde se ajustó el diseño de los planes de exploración vocacional y acompañamiento a la postulación, evaluando además la pertinencia de los objetivos, instrumentos de diagnóstico y metodologías de trabajo con estudiantes de $4^{\circ}$ año medio. De acuerdo a lo evaluado, se decidió implementar la actividad "Preparación Académica Temprana" en modalidad online, mediante un set de cuadernillos creados por los equipos de Lengua y Literatura, Matemáticas y Gestión Personal. Ello tuvo como finalidad repasar contenidos académicos, así como realizar acompañamiento socioemocional y vocacional, a través de un proceso individual guiado por las docentes de las asignaturas.

El área de Gestión Personal plantea su trabajo abordando dos unidades principales. La primera se encuentra enfocada en el proceso de autoconocimiento, a partir del análisis de características personales que permitan la conformación de un relato identitario, evaluando fortalezas, debilidades, reconociendo figuras de apoyo y episodios vitales que impliquen el desarrollo de la resiliencia. La segunda unidad aborda el área vocacional, mediante la exploración de la trayectoria de vida y la ejecución de un test vocacional, así como a través de mecanismos favorecedores de toma de decisiones, planteamiento de objetivos y la consecuente jerarquización de opciones, considerando elementos relevantes al momento de 
desarrollar una elección (duración y valor de la carrera, universidad y campus en que se imparte, acreditación, gratuidad, puntaje de corte, cupos PACE, campo laboral, entre otros).

\subsection{Participantes}

La actividad "Preparación Académica Temprana", en su versión online del año 2020, recibió la inscripción de 241 estudiantes de los doce establecimientos educacionales PACE-UACh de la Región de Los Lagos, pertenecientes a las comunas de San Pablo, San Juan de la Costa, Puyehue, Río Negro, Puerto Octay, Purranque y Frutillar.

\subsection{Instrumentos}

Al finalizar la actividad, las y los estudiantes dejaron registro de su experiencia en dos instrumentos de evaluación. El primero corresponde a la "Encuesta de satisfacción del programa", a través de la cual se advierte cuantitativamente la satisfacción general de las y los estudiantes y el aporte del área de Gestión Personal en materia de preparación académica temprana, en aspectos relacionados a autoconocimiento, ampliación de expectativas, toma de decisiones y construcción de proyectos de vida.

El segundo instrumento es el "Diagnóstico Final, Gestión Personal, Preparación Académica Temprana", donde es posible evidenciar de forma cualitativa los significados personales del proceso llevado a cabo por los y las estudiantes, junto al equipo del área de Gestión Personal.

\subsection{Procedimiento}

Durante el mes de mayo se realizó la impresión y entrega en formato físico de los cuadernillos de las tres asignaturas, a más de 200 estudiantes de sectores urbanos y rurales que declararon querer ser parte de esta iniciativa (El Llanquihue, 2020). Conjuntamente, se contactó a los/as estudiantes vía Whatsapp, formando grupos de no más de ocho personas, lo cual facilitaría 
el desarrollo de videollamadas a través de esta plataforma o Google Meet. A cada grupo se le asignaron docentes de Lengua y Literatura, Matemáticas y Gestión Personal, quienes distribuían las tutorías de acompañamiento en diversos días de una misma semana, con una frecuencia bisemanal y una duración de 45 a 60 minutos por asignatura. Todo ello se llevó a cabo entre los meses de junio y septiembre de 2020.

El acompañamiento en el área de Gestión Personal se inició mediante la aplicación de un diagnóstico socioemocional y vocacional, para posteriormente desarrollar las nueve actividades propuestas en el cuadernillo de trabajo.

De acuerdo al calendario de "Preparación Académica Temprana", cada actividad del cuadernillo de Gestión Personal debía ser entregada a la tutora responsable antes de las 23:59 horas de cada viernes, durante los tres meses que abarcó el trabajo a distancia. Las tutorías bisemanales desarrolladas en grupos fueron un espacio de reflexión de contenidos abordados individualmente. Esta instancia permitió el diálogo, la interacción con pares y la profundización en las experiencias de cada cual, brindando un espacio para realizar consultas, expresar preocupaciones y compartir aspiraciones de vida. 


\section{Tabla 1}

Resumen metodología para "Preparación Académica Temprana” (PAT), año 2020

\section{Etapa}

\section{Descripción}

Diseño

a) Revisión del Plan Operativo Institucional, ajuste de planes de exploración vocacional y acompañamiento a la postulación.

b) Planificación PAT online mediante cuadernillos de trabajo autónomo en Lengua y Literatura, Matemáticas y Gestión Personal.

c) Gestión Personal: trabajo en unidades de autoconocimiento y orientación vocacional.

Participantes

241 estudiantes de $4^{\circ}$ medio inscritos/as, pertenecientes a 12 establecimientos educacionales PACE-UACh.

Instrumentos - Encuesta de satisfacción del programa.

- Diagnóstico Final, Gestión Personal, Preparación Académica Temprana

Procedimiento a) Impresión y entrega a estudiantes de cuadernillos en formato físico. b) Implementación de grupos de Whatsapp (máximo 8 personas)

c) Tutorías por asignatura con duración de 45 a 60 minutos, cada dos semanas, entre los meses de junio y septiembre de 2020.

d) Implementación de diagnósticos inicial y final en Gestión Personal.

e) Desarrollo de 9 actividades totales, con entrega de reporte a tutora responsable antes de las 23:59 horas de cada viernes. 


\section{Resultados}

De un total de 241 estudiantes inscritos/as, 126 finalizaron el proceso exitosamente y 96 de ellos/as respondieron el instrumento "Encuesta de satisfacción del programa", entre el 31 de agosto y el 9 de septiembre de 2020. Dicho instrumento, de carácter anónimo, recogió la evaluación por parte de las/os estudiantes tanto de las áreas académicas como de Gestión Personal.

En relación a esta última área, fueron seleccionadas cinco preguntas claves que permiten dar cuenta del proceso de desarrollo socioemocional y fortalecimiento del autoconocimiento en estudiantes, orientados hacia la proyección de un plan de vida futuro.

En cuanto a la primera aseveración, "El programa me guió en la toma de decisiones", un $80,1 \%(\mathrm{~N}=77)$ consideró estar "de acuerdo o muy de acuerdo", mientras que un 15,6\% $(\mathrm{N}=$ 15) refirió estar "ni de acuerdo ni en desacuerdo". En tanto, un 4,2\% $(\mathrm{N}=4)$ señaló estar "en desacuerdo o muy en desacuerdo" con la afirmación.

Respecto a la segunda pregunta, "Las actividades ofrecidas aportaron a mi autoconocimiento", un 92,7\% (N=89) señaló "estar de acuerdo o muy de acuerdo" y solo un $5,2 \%(\mathrm{~N}=5)$ señaló estar "ni de acuerdo ni en desacuerdo". Por su parte, un 2\% $(\mathrm{N}=2)$ refirió estar "en desacuerdo o muy en desacuerdo".

En cuanto a la tercera pregunta, "El trabajo abordado en 'Preparación Académica Temprana' amplió las expectativas que tenía de mi futuro", un 86,4\% $(\mathrm{N}=83)$ señaló estar “de acuerdo o muy de acuerdo", un 11,5\% (N=11) "ni de acuerdo ni en desacuerdo", mientras que un $2 \%(\mathrm{~N}=2)$ afirmó estar "en desacuerdo o muy en desacuerdo".

En la cuarta pregunta, "El programa promovió la construcción de proyectos de vida", un $88,6 \%(\mathrm{~N}=85)$ afirmó estar "de acuerdo o muy de acuerdo", un 9,4\% $(\mathrm{N}=9)$ "ni de acuerdo ni en desacuerdo", mientras que un 2\% $(\mathrm{N}=2)$ señaló estar "en desacuerdo o muy en desacuerdo" con dicha afirmación. 
Respecto a la quinta pregunta, "El programa me permitió identificar con mayor claridad mis talentos", un 82,3\% ( $=79)$ mencionó estar "de acuerdo o muy de acuerdo" con la afirmación, mientras un 10,4\% $(\mathrm{N}=10)$ refirió estar "ni de acuerdo ni en desacuerdo" y un $7,3 \%(\mathrm{~N}=7)$ señaló estar “en desacuerdo o muy en desacuerdo" con la afirmación.

\section{Figura 1}

Valoración de áreas de acompañamiento en Gestión Personal, según preguntas

El programa me guió en la toma de decisiones

Las actividades aportaron a mi autoconocimiento

El trabajo abordado en PAT amplió las expectativas que tenía de mi futuro

El programa promovió la construcción de proyectos de vida

El programa me permitió identificar con mayor claridad mis talentos

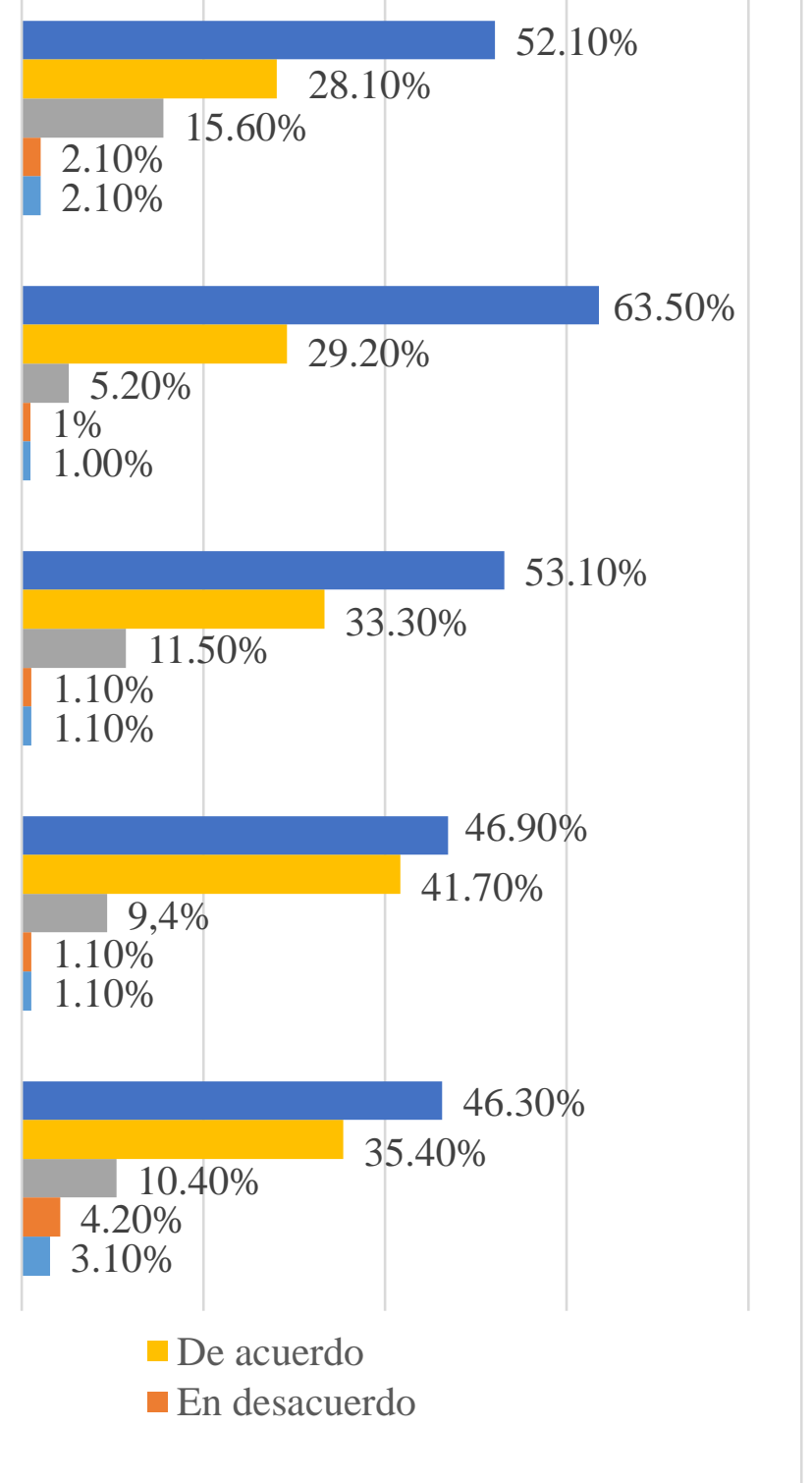

Muy de acuerdo

- Ni de acuerdo ni en desacuerdo - Muy en desacuerdo 
Respecto al grado de satisfacción general con el acompañamiento del Programa PACE, un 96,9\% ( $\mathrm{N}=93)$ declaró encontrarse "muy satisfecho/a o satisfecho/a", mientras que un $2,1 \%(\mathrm{~N}=2)$ se identificó con la opción "ni satisfecho/a ni insatisfecho/a". Solo un 1\% $(\mathrm{N}=$ 1) se mostró "muy insatisfecho/a" con la actividad.

\section{Figura 2}

Satisfacción general de estudiantes con el acompañamiento del Programa PACE-UACh

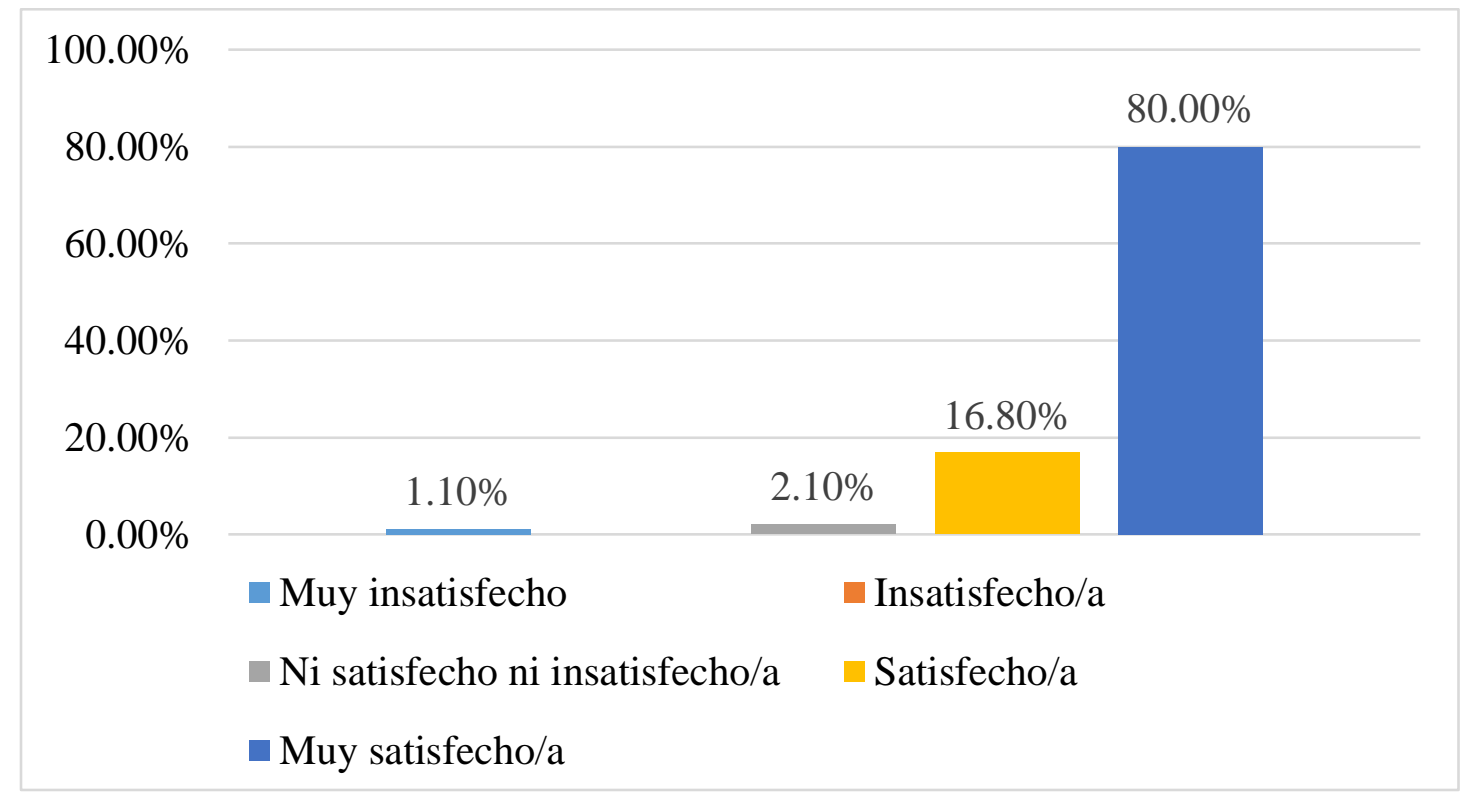

A través de las evaluaciones cualitativas del acompañamiento realizado en el área de Gestión Personal, es posible apreciar una positiva valoración tanto del trabajo desarrollado individualmente como de las tutorías e interacciones llevadas a cabo en este contexto. En las 126 respuestas obtenidas ${ }^{3}$ en el instrumento "Diagnóstico Final, Gestión Personal, Preparación Académica Temprana", se observa que los y las estudiantes destacan los significados personales del proceso a través de sus opiniones:

\footnotetext{
${ }^{3}$ La identidad de las y los estudiantes encuestados/as es resguardada mediante la identificación a través del número de la respuesta emitida.
} 
La verdad me ayudó mucho en tiempos de encierro. Cuando leía las actividades y al imaginar mi futuro y saber que podría lograrlo, me sentí con esperanza, me sentí en confianza y me fui conociendo a mí misma. Fueron mis actividades y clases favoritas. (Estudiante 52)

Me pareció como un psicólogo en un libro. Me sentí cómoda completándolo, me gustó su formato y me ayudó mucho a expandir mi visión hacia otras carreras y profundizar en las que me gustan. Nuestra orientadora me hizo sentir en un área segura y siempre respondió nuestras dudas con mucho cariño. Me gustó mucho estar en Gestión Personal y la profe me ayudó mucho con las dudas que tenía. (Estudiante 91)

En una línea similar, el estudiante 101 señala: "Para mí, Gestión Personal generó un proceso constructivo, ya que pude conocerme a mí mismo y valorar mis aptitudes, mis oportunidades y principalmente a mi familia. Todo esto contribuyó a que me sintiera un poco más feliz".

Por su parte, otra estudiante reflexiona

Considero que fue un espacio que necesitaba en el momento justo. Me ayudó enormemente a conocerme, a aprender de mí y de lo que me motiva, a liberar lo que me hacía daño, a apreciar lo que me hace bien. Desde el principio fue un espacio sano y seguro, nunca me sentí discriminada o apartada. Agradezco de todo corazón toda la ayuda que me dieron. (Estudiante 103)

Finalmente, el estudiante 124 sostiene: "Me ayudó a tener más claro lo que quiero para mi futuro, pensar más en mí, aclarar mis ideas y a no estresarme, y tomar todo con más calma".

Tanto las evaluaciones cuantitativas como cualitativas de estudiantes respecto al proceso de acompañamiento dan cuenta de una respuesta positiva a la actividad desarrollada, destacando el aporte del Programa a los procesos de toma de decisiones, ampliación de expectativas y construcción de proyectos de vida, así como también la identificación de 
talentos y características que potenciaron su proceso de autoconocimiento. El acompañamiento y soporte socioemocional en momentos de crisis, como el experimentado en el contexto actual, es ampliamente valorado por los y las estudiantes, al identificarlo como un espacio "sano y seguro", “de calma”, “[lo] que necesitaba en el momento justo”.

Lo anterior permite advertir lo importante y necesaria que resulta la implementación de acciones coordinadas, oportunas y efectivas en contexto educativo, ya sea en situaciones regulares o de emergencia, así como también en intervenciones futuras, ante un inminente regreso a las aulas.

\section{Discusión}

A nivel nacional, son diversas las instituciones de educación superior que han advertido la necesidad de modificar la metodología para ampliar el alcance de sus actividades en el contexto de emergencia sanitaria. Tal es el caso de las universidades adscritas al Programa PACE, quienes han desarrollado una serie de estrategias que les permiten acercar su contenido a las y los estudiantes.

En este contexto, el Programa PACE de la Universidad de Chile implementa desde el mes de mayo las Consejerías Virtuales, espacios de apoyo digital y personalizado para estudiantes de $3^{\circ}$ y $4^{\circ}$ medio, con el objetivo de entregar información, realizar acompañamiento y permitir la adquisición de herramientas por parte de las/os involucradas/os, favoreciendo una adecuada toma de decisiones.

Luego de dos semanas de su lanzamiento, la plataforma registraba más de 60 solicitudes de estudiantes requiriendo consejería virtual. Las principales demandas aludían a la obtención de información sobre el proceso de admisión 2021 (73\%), aclaración de dudas vocacionales (69\%), apoyo en la toma de decisiones (61\%) e información sobre el mundo laboral (56\%) (Universidad de Chile, 2020). Dentro de sus cifras destaca la consulta a un 12\% de estudiantes migrantes, siendo esta población y las acciones dirigidas a ella un aspecto relevante a considerar, al momento de diseñar acciones para estudiantes en contexto virtual. 
Por su parte, el Programa PACE y el Plan de Apoyo Estudiantil (PAE) de la Universidad Tecnológica Metropolitana (UTEM) destacan la labor realizada en contexto de emergencia, señalando que sus estrategias se generaron en conjunto con las comunidades educativas, levantando diagnósticos específicos, realizando catastros de la situación de los/as estudiantes y comunidades educativas. Este punto resulta relevante, ya que la realización de diagnósticos situados entrega una visión amplia de los contextos de las/os estudiantes y se convierte en un elemento de alto valor al momento de diseñar actividades y estrategias de acercamiento a las comunidades educativas.

La Universidad Tecnológica Metropolitana destaca la ejecución de estrategias de apoyo al bienestar socioemocional, mediante tutorías virtuales de orientación vocacional con un enfoque reflexivo y desde una perspectiva crítica. Las tutorías, según el equipo interventor, son una estrategia fundamental para mantener el vínculo del Programa con los/as estudiantes y, a la vez, de estos/as con sus propios procesos educativos (Universidad Tecnológica Metropolitana, 2020).

Con lineamientos comunes a los seguidos por las universidades anteriormente mencionadas, y tal como se expuso en los resultados, el Programa PACE de la Universidad Austral, sede Puerto Montt, corrobora la importancia de los procesos de acompañamiento vocacional y emocional en contexto de crisis.

Las estrategias de acompañamiento llevadas a cabo por esta institución favorecen el desarrollo de un proceso continuo, posible de anticipar y programar, en un escenario adverso de alta incertidumbre, entregando un apoyo constante y sostenido. Del mismo modo, la interacción planificada a través del sistema de tutorías formaliza un canal de comunicación directo entre las profesionales y las y los estudiantes, permitiendo la expresión de dudas vocacionales y emocionales con una mayor y más rápida capacidad de respuesta.

La implementación de grupos de trabajo pequeños con personas que se conocen entre sí, facilitó la creación de un espacio seguro y cercano, siendo esto reforzado en las respuestas de las y los estudiantes. Ello permitió reflexionar sobre los contenidos con mayor profundidad y plantear discusiones espontáneas sobre temas propuestos por las/os 
participantes en las tutorías, tales como el adultocentrismo, la evaluación de redes de apoyo familiar, la capacidad de tomar decisiones, el proceso de independización y vida fuera del hogar, el reconocimiento de emociones, estrategias para manejar el estrés y la ansiedad, entre otros.

En el transcurso del proceso, fue posible advertir que la utilización de esta metodología se encuentra restringida por las posibilidades de los y las estudiantes de acceder a un dispositivo y conexión a internet, lo cual en muchos casos es inexistente. Pese a los intentos por asegurar la conectividad y participación, a través del aumento de los canales de comunicación vía Whatsapp, llamadas telefónicas y servicios de videollamada de menor uso de datos móviles, quedó en evidencia la dificultad que tienen muchos y muchas estudiantes para recibir las propuestas educativas destinadas a ellos/as, revelando una nueva brecha en términos de conectividad, que refleja limitaciones geográficas y socioeconómicas.

En este sentido, una de las principales limitaciones del presente estudio se encuentra en la diferencia existente entre el número de estudiantes inscritos/as inicialmente en la actividad y la cantidad de quienes finalizan el proceso de manera exitosa, que se reduce a casi la mitad. Si bien año a año esta actividad advierte una baja en el número final de participantes respecto al inicial, resultaría interesante analizar de qué modo la situación de emergencia sanitaria afectó la participación de las y los estudiantes.

En este punto es importante advertir que la inversión en recursos humanos y materiales que realiza este tipo de programas ministeriales es lo que posibilitó, en el caso del PACEUACh, la implementación del proceso de acompañamiento detallado en este documento. Es fundamental que exista un mayor compromiso con la inyección de recursos estatales en materia de educación, para que así todos los establecimientos, independientemente de su ubicación, puedan contar con las condiciones materiales y humanas necesarias para que cada estudiante acceda a la educación en igualdad de condiciones.

En un mundo globalizado como el nuestro y con las demandas que se presentan hoy en día, la conectividad se plantea como un gran desafío. Asegurar el acceso transversal a dispositivos electrónicos y a una adecuada conexión debe ser un derecho fundamental que 
permita a los y las estudiantes un acceso equitativo a la educación, independientemente de su situación geográfica y socioeconómica.

\section{Conclusiones}

Para el equipo PACE-UACh de la Región de Los Lagos, la puesta en marcha de la actividad y la metodología señalada posibilitó el análisis y la reestructuración de estrategias y actividades que permitiesen continuar trabajando para cumplir los objetivos propuestos. La emergencia sanitaria desafió las lógicas de lo presencial y los límites de la educación tradicional, transformando muchas prácticas impensadas en una realidad.

Al inicio del presente artículo, se planteó la interrogante respecto a la posibilidad de ampliar expectativas y fortalecer los procesos de desarrollo socioemocional y vocacional en las/os estudiantes, en contexto de virtualidad y emergencia sanitaria. En ese marco, los resultados obtenidos nos permiten reflexionar respecto a los alcances y significados que el uso de esta metodología tuvo para las y los estudiantes, pudiendo apreciarse la alta valoración de la experiencia y el consecuente logro del objetivo del Área de Gestión Personal, según lo planteado en su Programación Operativa Institucional 2020.

Desde la perspectiva de la investigadora y ejecutora de la actividad, la realización de esta metodología de trabajo promovió indirectamente en los y las estudiantes la adquisición de habilidades actitudinales, tales como autodisciplina, proactividad, responsabilidad y compromiso. Del mismo modo, se advierte la participación e involucramiento en sus propios procesos educativos, al ser ellos/as quienes señalan el aporte significativo del programa para la construcción de un proyecto de vida, el reconocimiento de sus talentos y la ampliación de expectativas, reforzando su proceso de autoconocimiento y su capacidad para tomar decisiones. 
Aún se desconocen los alcances de la pandemia, tanto en términos económicos, políticos, educativos, como en materia de salud mental de los/as adolescentes. En este sentido, la implementación de estrategias y acciones que aborden el área socioemocional en todos los niveles educativos, resulta imprescindible como una forma de reducir el impacto negativo que esta situación pueda tener en la salud mental y las expectativas de futuro de los/as estudiantes.

La implementación del acompañamiento socioemocional no puede ser una opción, así como tampoco puede esperar el regreso a las clases presenciales, considerando la diversidad de contextos sanitarios enfrentados a lo largo del país. Responder a esta necesidad es un deber ético de gran relevancia y, por lo mismo, la propuesta aquí planteada se transforma en la manifestación de una buena práctica que, puesta a disposición, puede ser replicada en otros contextos, considerando sus particularidades tanto culturales como territoriales.

\section{Referencias}

Bisquerra, R., y Pérez, N. (2007). Las competencias emocionales. Educación XXI, 10, 61-82. http://e-spacio.uned.es/fez/eserv/bibliuned:EducacionXXI-2007numero10823/Documento.pdf

Deutsche Welle. (2020). (08 de mayo de 2020). Escuelas en Alemania en la crisis del coronavirus. Todo a distancia, pero lejos de la normalidad. https://www.dw.com/es/escuelas-en-alemania-en-la-crisis-del-coronavirus-todo-adistancia-pero-lejos-de-la-normalidad/a-53374147

Educación 2020. (23 de abril de 2020). Encuesta Educación 2020 y estado de ánimo de estudiantes ante la pandemia: 63\% se siente aburrido y solo un 3\% está "feliz" en casa. http://educacion2020.cl/noticias/encuesta-educacion-2020-y-estado-de-animo- 
de-estudiantes-ante-la-pandemia-63-se-siente-aburrido-y-solo-un-3-esta-feliz-encasa/

El Llanquihue. (01 de septiembre de 2020). Programa PACE de la UACh Puerto Montt apoya a más de 200 estudiantes de educación media. https:/www.soychile.cl/Puerto-Montt/Sociedad/2020/09/01/671398/ProgramaPace-de-la-UACh-Puerto-Montt-apoya-a-mas-de-200-estudiantes-de-educacionmedia.aspx?fbclid=IwAR186wLIuo6VgP0KU1xcInWvz9GF3KSECh_n_k4T2nTr2AMF_lqb0oL1b8

Ministerio de Educación. (s.f.). ¿Qué es el PACE? https://pace.mineduc.cl/sobre-elprograma-pace/que-es-el-pace/

Ministerio de Educación. (2015). Aprendizajes vitales: formación socioemocional desde los sectores de aprendizaje. https://unesdoc.unesco.org/ark:/48223/pf0000244681

Ministerio de Educación. (08 de mayo de 2020a). Aprendizaje socioemocional en tiempos de pandemia: Mineduc, Unesco y Unicef se unen para educar sobre impacto socioemocional en comunidades educativas por pandemia de coronavirus. https://www.mineduc.cl/aprendizaje-socioemocional-en-tiempos-de-pandemia/

Ministerio de Educación. (29 de mayo de 2020b). Apoyo socioemocional en tiempos de pandemia: Mineduc entrega recomendaciones para el apoyo socioemocional de docentes, estudiantes y sus familias. https:/www.mineduc.cl/apoyo-socioemocionalen-tiempos-de-pandemia/

Universidad de Chile. (13 de mayo de 2020). Universidad de Chile lanza espacio de orientación para estudiantes de enseñanza media. https://www.uchile.cl/noticias/163363/nuevo-espacio-de-orientacion-paraestudiantes-de-ensenanza-media 
Universidad Tecnológica Metropolitana. (27 de mayo de 2020). La importante labor del Programa PACE y PAE en tiempos de emergencia sanitaria. https://noticias.utem.cl/2020/05/27/la-importante-labor-del-programa-pace-y-paeen-tiempos-de-emergencia-sanitaria/ 